\title{
Accuracy to detection timing for assisting repetitive facilitation exercise system using MRCP and SVM
}

\author{
Satoshi Miura1*, Junichi Takazawa², Yo Kobayashi ${ }^{3}$ and Masakatsu G. Fujie ${ }^{3}$
}

\begin{abstract}
This paper presents a feasibility study of a brain-machine interface system to assist repetitive facilitation exercise. Repetitive facilitation exercise is an effective rehabilitation method for patients with hemiplegia. In repetitive facilitation exercise, a therapist stimulates the paralyzed part of the patient while motor commands run along the nerve pathway. However, successful repetitive facilitation exercise is difficult to achieve and even a skilled practitioner cannot detect when a motor command occurs in patient's brain. We proposed a brain-machine interface system for automatically detecting motor commands and stimulating the paralyzed part of a patient. To determine motor commands from patient electroencephalogram (EEG) data, we measured the movement-related cortical potential (MRCP) and constructed a support vector machine system. In this paper, we validated the prediction timing of the system at the highest accuracy by the system using EEG and MRCP. In the experiments, we measured the EEG when the participant bent their elbow when prompted to do so. We analyzed the EEG data using a cross-validation method. We found that the average accuracy was $72.9 \%$ and the highest at the prediction timing $280 \mathrm{~ms}$. We conclude that $280 \mathrm{~ms}$ is the most suitable to predict the judgment that a patient intends to exercise or not.
\end{abstract}

Keywords: Neurorehabilitation, Repetitive facilitation exercise, Brain-machine interface, Motor command detection

\section{Background}

The number of cerebral stroke patients is increasing worldwide. For example, in Japan, cerebral stroke patients exceeded 2.8 million people in 2015 [1]. Patients often suffer from aftereffects following a stroke, the most frequent of which is hemiplegia. To recover motor function following hemiplegia, patients must endure a long course of difficult rehabilitation. Many studies have investigated methods to shorten the recovery time through efficient rehabilitation after hemiplegia $[2,3]$.

Neurorehabilitation has been shown to be very efficient $[4,5]$. Neurorehabilitation is a method to prompt the recovery of the injured neural system. Repetitive facilitation exercise is drawing attention as a particularly effective rehabilitation. Kawahira demonstrated the efficacy of

\footnotetext{
*Correspondence: miura.s@aoni.waseda.jp

1 Faculty of Science and Engineering, Waseda University, 3-4-1, Okubo,

Shinjuku-ku, 169-8555 Tokyo, Japan

Full list of author information is available at the end of the article
}

repetitive facilitation exercise [6]. Patients using repetitive facilitation exercise can recover motor control three times faster than those using usual therapy [7]. Repetitive facilitation exercise also improves the paralysis part to health compared with usual therapy [8].

Figure 1 shows the mechanism of the repetitive facilitation exercise. The patient imagines moving a paralyzed body part. Within the patient's body, motor commands travel from the brain, through the spinal cord, to the paralyzed part. The therapist then stretches the paralyzed part of the patient using physical or electrical stimulation before the motor command reaches the spinal cord. This stimulation excites the nerves in the spinal cord and activates the path of the motor command. Because the motor command can pass more easily through the nerve pathway, the patient becomes likely to regain the ability to move the paralyzed part unaided.

The most important point of repetitive facilitation exercise is the stimulation timing. It is necessary for the stimulation to occur before the motor command reaches 


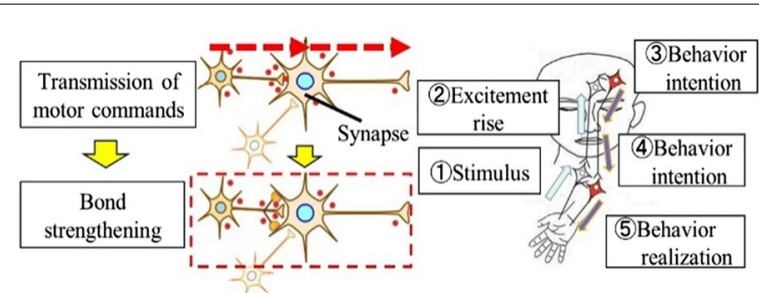

Fig. 1 Principle of repetitive facilitation exercise. When a motor command occurs in the brain, the synapse bond strengthens. The neural networks are excited. At this time, the therapist stimulates the patient's paralyzed arm. This stimulation is transmitted to the brain. When the intention to move the arm and the stimulus overlaps, the brain recognizes this as if the patient had moved the paralyzed hand. This recognition promotes the reconstruction of the nerve pathway to the paralyzed arm

the spinal cord for success of the repetitive facilitation exercise. However, it is difficult to successfully perform repetitive facilitation exercise because even a skilled therapist cannot detect the timing of the motor command. The success of repetitive facilitation exercise is dependent on the intuition and experience of the therapist.

Even an experienced therapist cannot achieve a 100\% success rate with the repetitive facilitation exercise because therapists cannot detect patient motor intentions. It has been reported that five of twelve patients showed increased motor evoked potential after repetitive facilitation exercise by therapists [21]. This shows that the nervous systems of these patients underwent some reconstruction. Although there are individual differences, it is said that the conventional success rate is about five of twelve, that is just $45 \%$. To improve the higher success rate than the target value $45 \%$, therapists require a system to assist repetitive facilitation exercise. An upper limb reaching device has been proposed to reduce fatigue and pain in the paralyzed arm and to decrease the burden on the therapist [9]. The patient repeats inward and outward movements to push the front and back buttons alternately. When the patient pushes the button, the device generates vibrations and electric stimulation to make it easier to move the paralyzed arm. However, the device cannot control the accurate stimulation timing because the device cannot detect the motor command generated in the brain.

\section{Related work}

There have been many studies of real time detecting the motor command in brain. Most of these have used electroencephalogram (EEG) because it has a higher time resolution than other brain measurement devices. EEG is simple to analyze in real time. For example, Lucian reported that EEG can show the steering timing of a driver during driving $[10,11,12]$.
He measured and analyzed EEG data while participants operated a driving simulator. Using this method, the turn direction was detected $811 \mathrm{~ms}$ before steering with an accuracy of $74.6 \%$. In another study, Choi showed that a brain-machine interface system using EEG could be used to control a wheelchair [13]. This system analyzed EEG data and moved forward or turned left or right based on the measured EEG signals within $125 \mathrm{~ms}$.

These studies are useful for realizing real-time brainmachine interfaces for healthy individuals. However, these studies have not been adapted to the rehabilitation.

\section{Objective}

Our motivation is to develop a brain-machine interface system to assist repetitive facilitation exercise. The system detects motor intention in real time by measuring the brain and stimulates the paralyzed body part before the motor command reaches the spinal cord, as shown in Fig. 2. The system overview and flow are shown in Figs. 3 and 4. The system is consisted of the EEG measurement device and FES generator, shown in Fig. 3. The system detects motor commands from the patient's EEG data using support vector machine (SVM). It then stimulates the paralyzed part by functional electric stimulation (FES) before the motor command reaches the spinal cord. The system provides stimulation at the same time the SVM detects the motor command; thus, the stimulation

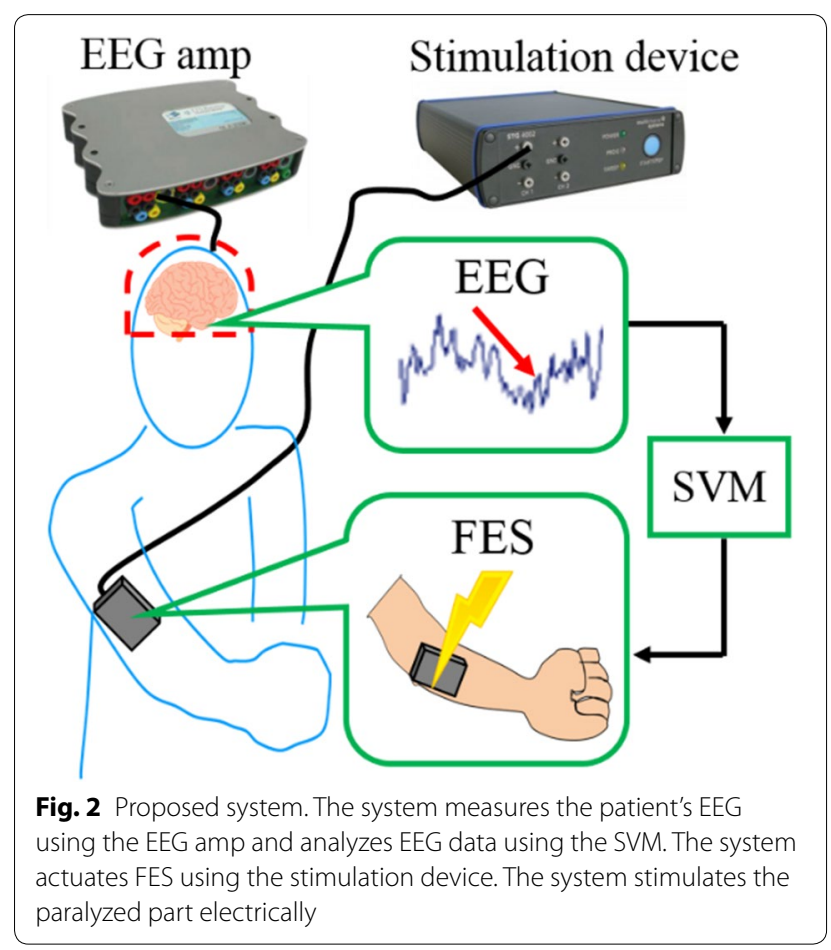




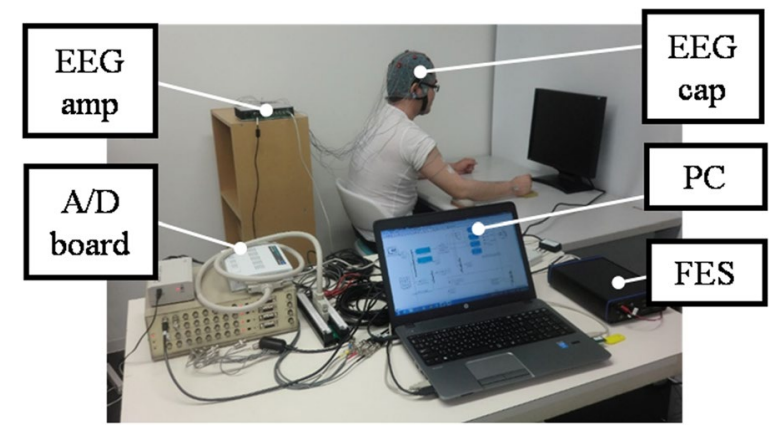

Fig. 3 Overview of the system. An EEG cap is set on the patient's head. EEG data are measured by the EEG cap, amplified by the EEG amp and output via the A/D board to a PC. The PC is used to analyze the EEG data and actuate the FES

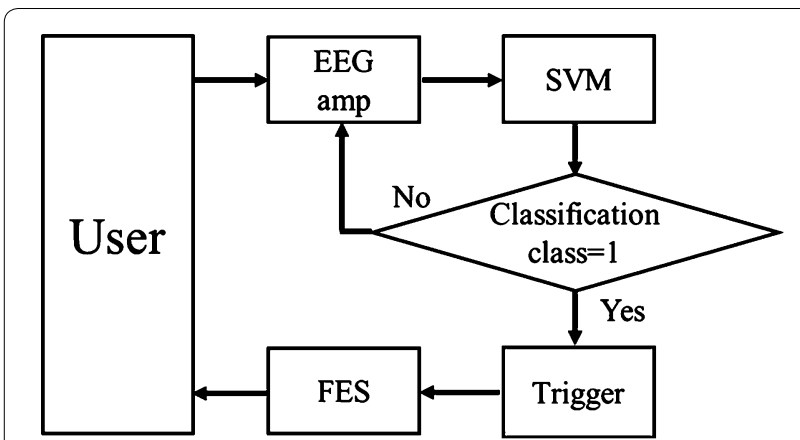

Fig. 4 Flow of the system. The system measures the patient's EEG data and analyzes it using SVM. If classification class by SVM is 1, the trigger is on and the system actuates the FES to stimulate the patient

occurs after the motor command is produced, but before it reaches the spine.

In this paper, we validate our proposed motor command detecting method. The prediction accuracy changes as to the timing before action. It is not clear that the accuracy changes as to the prediction timing changing. We clarify the appropriate prediction timing by the accuracy of the system using SVM. We constructed an SVM system and carried out experiments to clarify the detection ratio of motor commands. In the experiment, we collected EEG data when the participant bent their elbow, shown in Fig. 5. To facilitate the timing, we displayed a bar on a monitor to indicate to the participant when to bend their elbow. The bar was displayed on the monitor and shortened gradually until it disappeared. At the same time the bar disappeared, the participant had to bend their elbow. We analyzed EEG data using a cross-validation method to clarify the detection ratio of the motor command. We confirmed that the calculated detection ratio was above the target value, verifying the utility of the proposed system.

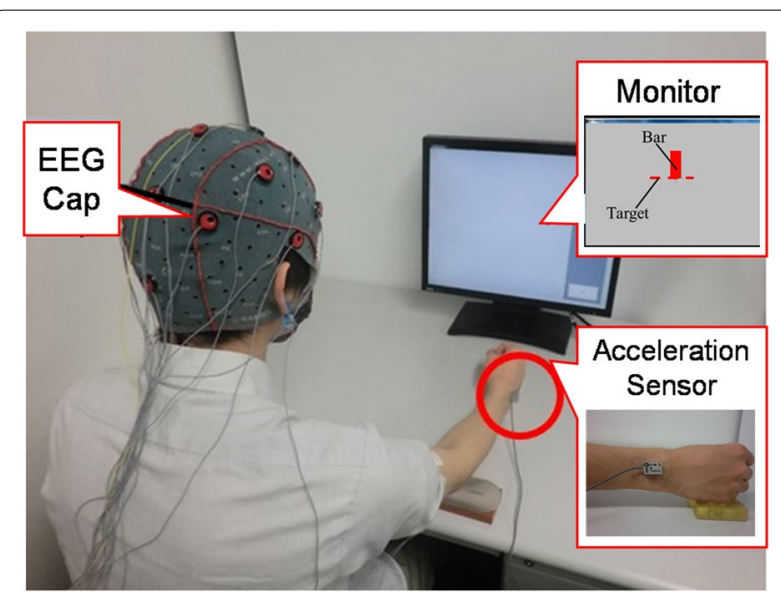

Fig. 5 Experimental overview. The EEG cap is set on the participant's head, and the accelerometer is put on the participant's wrist. First, the participant remains relaxed. A red bar is displayed on the monitor and shortens gradually. At the same time the bar disappears, the participant bends their elbow. We detect when the participant bends the elbow by analyzing the acceleration of the wrist

\section{Methods \\ Participants}

Three healthy participants (male, age 22-23, two righthanded and one left-handed) were enrolled in the experiment. We did not enroll paralyzed patients because the aim of the present study was only to validate the proposed method for detecting motor commands. Informed consent was obtained from all participants. All participants attested to having slept well the night before the experiment to exclude the influence of sleep deprivation. All participants did not intake the drugs such as caffeine, alcohol, nicotine, and other medicines. The experiments were approved by the Waseda University Institutional Review Board (No. 2014-156).

\section{Experimental setup}

We used an EEG (g. USBamp, gtec, USA) to measure brain activity. We set up the device as shown in Table 1 . The sampling frequency was set to $256 \mathrm{~Hz}$ because the real-time measurement using MRCP needs a high temporal resolution. We used 17 analog input channels and 1 GND passive channel. On an EEG cap, 14 channels were located to measure EEG based on the international 10-20 system, as shown in Fig. 6. The reference electrode was put on the earlobe.

We used a three-degree-of-freedom accelerometer (ACL300, Biometric Inc., USA) to detect the time when the participant moved their arm. We affixed the accelerometer to the participant's wrist as shown in Fig. 6 and connected it to an analog input-output board (AIO-163202FX-USB, Contec Inc., USA) to get the analog input value. The accelerometer used three channels, as shown in Table 1. 
Table 1 Device settings of a g. USBamp and ACL300

\begin{tabular}{lll}
\hline Title & Specification & Mounting position \\
\hline Sampling rate Hz & 256 & \\
Analog input channel ch & 17 & F7 \\
Mounting position & ch1 & F3 \\
& ch2 & Fz \\
& ch3 & F4 \\
& ch4 & F8 \\
ch5 & T7 \\
ch6 & C3 \\
ch7 & Cz \\
ch8 & C4 \\
ch9 & T8 \\
ch10 & P3 \\
ch11 & Pz \\
ch12 & P4 \\
ch13 & Oz \\
ch14 & Acceleration sensor ch1 \\
ch15 & (X-axis) \\
ch16 & Acceleration sensor ch2 \\
& (Y-axis) \\
ch17 & Acceleration sensor ch3 \\
& (Z-axis) \\
GND & Nz \\
\hline
\end{tabular}

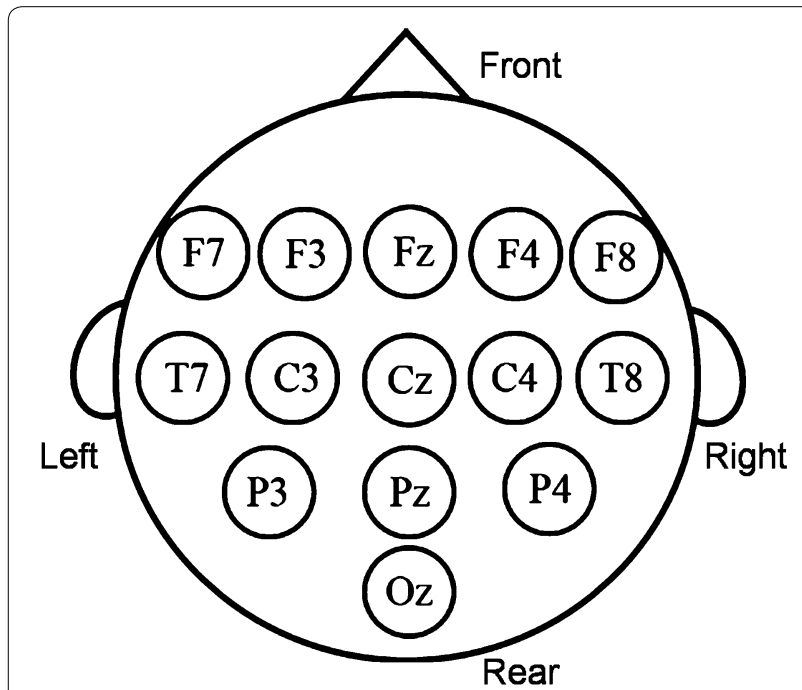

Fig. 6 Measured points on the EEG cap, viewed from directly above the patient's head. The 14-ch EEG data are measured at these points

\section{Experimental task}

We conducted the experiment in a closed room to minimize noise disturbances. During the experiment, the participant did not talk and sat still. In addition, we asked the participant to try to avoid swallowing saliva or blinking hard. The temperature was $20 \pm 15^{\circ} \mathrm{C}$, and the humidity was $45-85 \%$.

We put the EEG cap and the accelerometer on the participant. The experimental procedure is shown in Fig. 8. In the initial state, the participant relaxed with their right forearm resting on the desk, palm up. One measurement session consisted of rest, preparation and act periods within $10 \mathrm{~s}$. During the rest period, from 0 to $3 \mathrm{~s}$, the participant relaxed and looked at the monitor. During the preparation period, from 3 to $6 \mathrm{~s}$, a red bar was displayed on the monitor and became smaller until disappearing at $6 \mathrm{~s}$, as shown in Fig. 7. During the act period, after $6 \mathrm{~s}$, at the same time as the red bar disappeared, the participant bent their arm at the elbow. The participant kept their elbow bent for approximately $0.5 \mathrm{~s}$ and then set their arm to the initial resting state. This experimental procedure was performed 100 times by each participant.

\section{Experimental condition}

The experimental condition is the prediction timing. The prediction accuracy changes as to the timing before

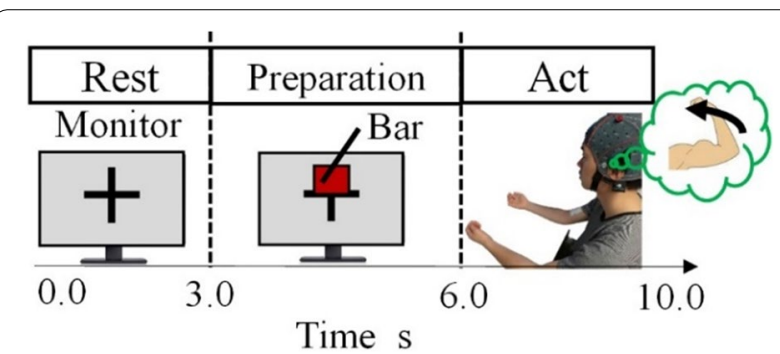

Fig. 7 One measurement session. The rest period is $0-3 \mathrm{~s}$ from starting measurement. The preparation period is $3-6 \mathrm{~s}$ and includes the appearance, gradual shortening and disappearance (at $6 \mathrm{~s}$ ) of the red bar. The act period is $6-10 \mathrm{~s}$, beginning at the same time as the bar disappears. In the act period, the participant bends their elbow

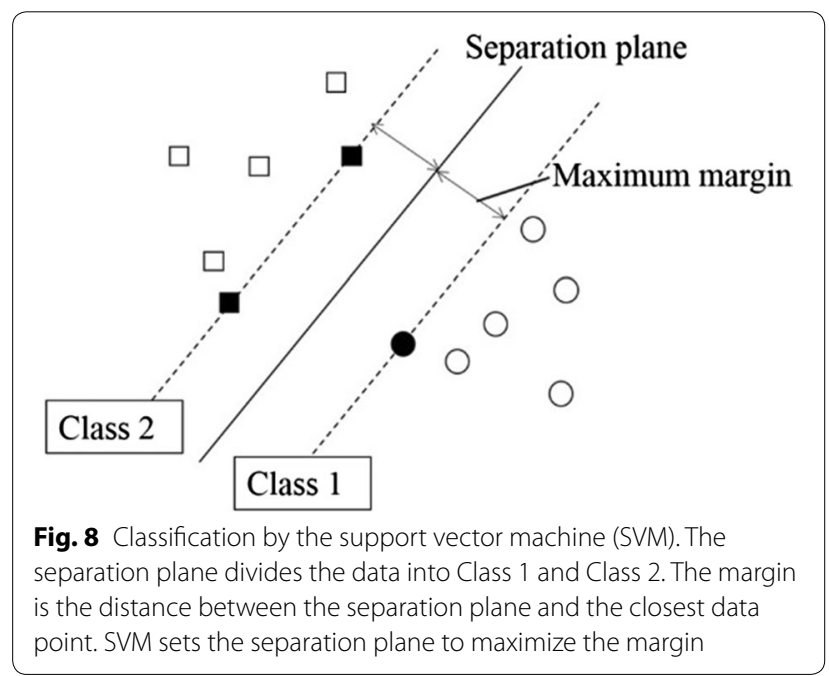


action. However, the timing is influenced by the recognition delay. Even the participant bent his/her elbow as soon as the disappearance of the bar, and there is actually the delay because it takes time to recognize it. The recognition delay is said to about $200 \mathrm{~ms}$, but there is no clarity of accurate delay. In this paper, the experimental condition is the prediction timing around $200 \mathrm{~ms}$ before action timing. The condition is $70,140,210,280$ and $350 \mathrm{~ms}$ before action timing.

\section{Analysis \\ Movement-related cortical potential (MRCP)}

We focused on the functions of EEG. For example, event-related potential is the electric fluctuation detected from neurons following light or sound stimulus [14]. The P300 speller, a communication device for severely paralyzed patients, utilizes the event-related potential function. Event-related desynchronization is another function in which the power spectrum of the EEG alpha band decreases following motor commands [15]. Event-related desynchronization is often used in rehabilitation systems. In the present study, we used movement-related cortical potential (MRCP). MRCP is the change in EEG signal resulting from the plan and action of voluntary exercise $[16,17]$. MRCP is detectable before and after exercise. In particular, MRCP that starts about $800 \mathrm{~ms}$ before exercise is called the motor readiness potential. We hypothesized that the motor readiness potential would show the timing of when a motor command occurs in the brain.

\section{Support vector machine (SVM)}

To detect MRCP, a pattern identification unit is required. There are two kinds of pattern identification unit: One uses a parametric method for which the probability distribution of data is known in advance and another uses a nonparametric method which requires collected data because the probability distribution of data is unknown. We used the nonparametric method because EEG data are different for each patient.

We employed a support vector machine (SVM) because it can divide known data into two classes [18, 19, 20]. Compared with other algorithms, SVM is suitable to judge the two classes that the human tends to move his body or not. Using this SVM system, we divided the EEG data into data during rest and data during action. The system needs to detect EEG data during action as a motor command.

SVM is a supervised learning method that can construct pattern identification to two classes. SVM learns the parameters required to maximize the margin from training sample data. SVM decided the two outputs using the following:

$$
y=\operatorname{sign}\left(W^{T} x-h\right)
$$

where $W$ is the weight parameter and $h$ is the threshold. If $u>0, \operatorname{sign}(u)$ is 1 . If $u \leq 0, \operatorname{sign}(u)$ is -1 . Figure 7 shows the SVM classification. The Class 1 and Class 2 mean that the label of each class is 1 and -1. The margin is the distance between the separation plane and the closest data point. SVM finds an optimal value of $W$ to maximize the margin.

\section{Judgment of movement from acceleration}

We recorded the time when the participant bent their elbow using the accelerometer. We calculated the following:

$$
V_{x y z}=\sqrt{V_{x}^{2}+V_{y}^{2}+V_{z}^{2}}-0.1
$$

where $V_{x y z}$ is the combined acceleration, and $V_{x}, V_{y}$ and $V_{z}$ are the $\mathrm{X}$-, $\mathrm{Y}$ - and $\mathrm{Z}$-axis components of the acceleration. We clarified the maximum value of the acceleration during the rest period. We set the maximum acceleration value during rest period as the threshold of the movement starting judge. We judged when the acceleration was over the threshold as the timing when the participant bent the elbow.

To detect motor commands, we used EEG data from 0 to $2 \mathrm{~s}$ during each measurement session as the feature quantity during rest. EEG data at $210 \mathrm{~ms}$ after the acceleration of the wrist were considered the threshold for motor command. There are two reasons to set this threshold to $210 \mathrm{~ms}$ : One is the human cognitive delay. In this experiment, the participant bent and their elbow based on a signaling displayed on a monitor-the disappearance of the red bar. Therefore, we considered that it would take $200 \mathrm{~ms}$ after the bar disappears for the participant to recognize the bar disappearance. Another reason is machine delay. There is a machine delay of $10 \mathrm{~ms}$ from motion intention detection by the SVM to actuation of the FES. From the above, we set the expected delay to $210 \mathrm{~ms}$.

We clarified the identification ratio using cross-validation of the data from 100 trials by each participant. Figure 9 shows the cross-validation method. We divided the original data into $k$ blocks. Using the first block as test data and the other data as training data, we calculated the discrimination ratio. Next, using the second data as test data and the other data as training data, we calculated the discrimination ratio again. By repeating the above procedure $k$ times, we used the average of $k$ 


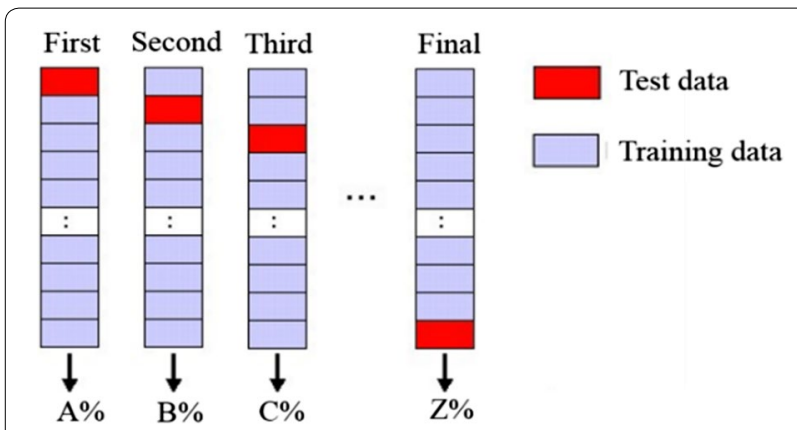

Fig. 9 Cross-validation method. The red blocks represent test data. The purple blocks represent training data. First, we considered the first data block as test data, and all other data blocks training data. Second, we considered the second data block as test data, and all other data blocks training data. We repeated this procedure $k$ times

values for discrimination rate to estimate the accuracy of the model.

Figure 10 shows the procedure for constructing the SVM. The action time is the timing when the acceleration of the wrist was over the maximum during rest. The detection time is $210 \mathrm{~ms}$ before the action time. We selected a value for $k$ of 10 , and the feature quantity was all 14-ch EEG data during $210 \mathrm{~ms}$ from the detection time to the action time.

To compare with other detection timings, we validate the discrimination rate by each $70 \mathrm{~ms}$ via cross-validation. The condition is 70, 140, 210, 280, $350 \mathrm{~ms}$.

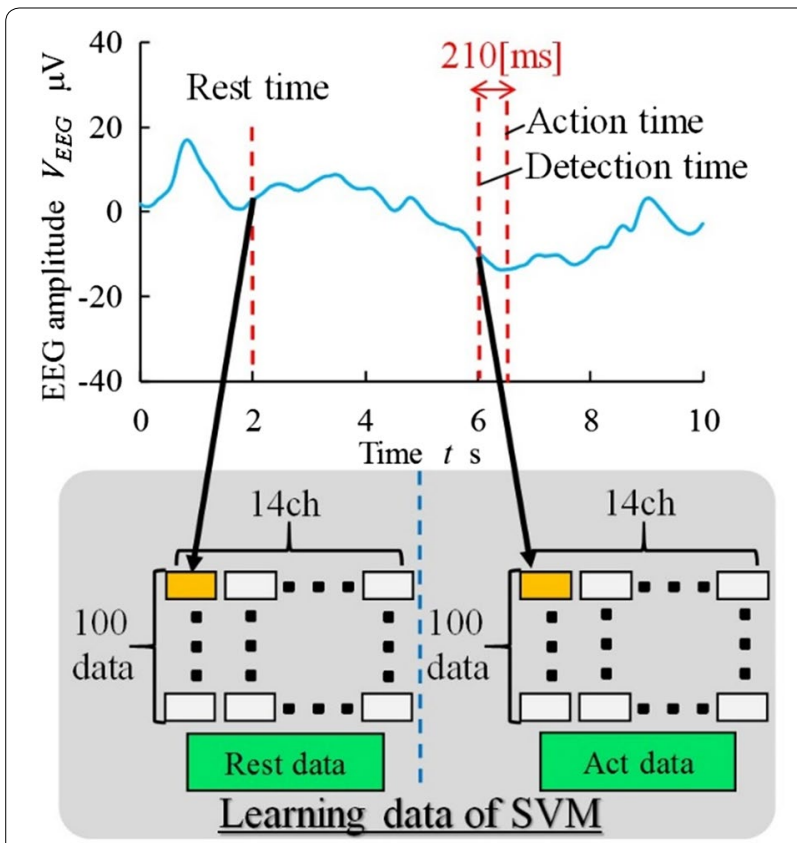

Fig. 10 Construction method of the SVM. The graph above is a sample of EEG data. SVM learns the data during rest and act periods

\section{Results and discussion}

Discrimination ratio by each detection timing is shown in Table 2. This discrimination ratio is about $70 \%$. The results show that SVM could detect the MRCP effectively. Particularly, the highest average is $72.9 \%$ at $280 \mathrm{~ms}$ so we determine that the most appropriate detection timing is $280 \mathrm{~ms}$.

We clarified the discrimination ratio at $280 \mathrm{~ms}$ for each participant, as shown in Fig. 11. The discrimination ratios of participants A, B and C were $67.5,69.4$ and $81.9 \%$, respectively. The average was $72.9 \%$; this is over the $45 \%$ target value.

Using EEG data $210 \mathrm{~ms}$ after the red bar disappeared, all discrimination data were at least $67 \%$. This result was above the $45 \%$ target value. This indicates that using EEG data sorted by SVM, the proposed system can perform FES on paralyzed patients with adjustable timing. Repetitive facilitation exercise administered using the proposed EEG system is potentially more successful than that administered by a therapist.

In the present study, we carried out the experiment by only three participants. We should conduct experiments using more participants. In addition, the EEG data were collected from only healthy subjects. For some stroke patients, although the neural system is different from the healthy subject, the sensory recognition motor loops would be same as to healthy because the neural system cannot feedback but can feedforward. We should validate the detection using EEG signals of paralyzed patients compared with the healthy people. In future work, we will develop the system using FES.

Table 2 Experimental result of detection rate

\begin{tabular}{|c|c|c|c|c|c|}
\hline \multirow[t]{2}{*}{ Subject name } & \multicolumn{5}{|c|}{ Detection rate for shift time $r \%$} \\
\hline & $70 \mathrm{~ms}$ & $140 \mathrm{~ms}$ & $210 \mathrm{~ms}$ & $280 \mathrm{~ms}$ & $350 \mathrm{~ms}$ \\
\hline Average all subjects & 70.9 & 72.0 & 71.8 & 72.9 & 72.5 \\
\hline Subject A & 69.0 & 68.0 & 69.0 & 67.5 & 73.5 \\
\hline Subject B & 65.6 & 69.9 & 69.4 & 69.4 & 65.1 \\
\hline Subject C & 78.2 & 78.2 & 77.1 & 81.9 & 79.3 \\
\hline
\end{tabular}

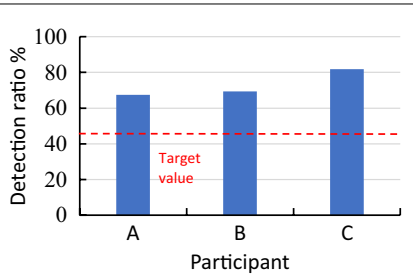

Fig. 11 Detection rate for each subject. The discrimination ratios of participants A, B and C are 69, 69.4 and $77.1 \%$, respectively. The target value is $45 \%$ 


\section{Conclusions}

In the present study, we proposed a brain-machine interface system to assist repetitive facilitation exercise. As a result, the average accuracy was $72.9 \%$ and the highest at the prediction time $280 \mathrm{~ms}$. We conclude that $280 \mathrm{~ms}$ is the most suitable to predict the judgment that a patient intends to exercise or not. In future work, we will develop this repetitive facilitation exercise assistance system.

\section{Authors' contributions}

SM conceived the study and drafted the manuscript. JT carried out all experiments and analyzed the data. KY and MF participated in the research design and sequence alignment. All authors read and approved the final manuscript.

${ }^{1}$ Faculty of Science and Engineering, Waseda University, 3-4-1, Okubo, Shinjuku-ku, 169-8555 Tokyo, Japan. ${ }^{2}$ Graduate School of Science and Engineering, Waseda University, 3-4-1, Okubo, Shinjuku-ku, 169-8555 Tokyo, Japan. ${ }^{3}$ Healthcare Robotics Institute, Future Robotics Organization, Waseda University, 3-4-1, Okubo, Shinjuku-ku, 169-8555 Tokyo, Japan.

\section{Acknowledgements}

The authors of this paper would like to thank Waseda University in Japan by offering the funding support for this publication.

\section{Competing interests}

The authors declare that they have no competing interests.

\section{Availability of data and materials}

The datasets supporting the conclusions of this article are included within the article.

\section{Funding}

This research was supported in part by the Outstanding Graduate COE Support Subsidy "Global Robot Academia (GRA)" from MEXT; in part by JSPS KAKENHI under Grant Numbers JP16H07265, JP14J07226, 26242061; and in part by the Council for Science, Technology and Innovation (CSTII), Cross-ministerial Strategic Innovation Promotion Program (SIP) (funding agency: JST).

\section{Publisher's Note}

Springer Nature remains neutral with regard to jurisdictional claims in published maps and institutional affiliations

Received: 18 August 2017 Accepted: 31 October 2017

Published online: 07 November 2017

\section{Author details}

6. Kawahira K, Shimodozono M, Ogata A, Tanaka N. Addition of intensive repetition of facilitation exercise to multidisciplinary rehabilitation promotes motor functional recovery of the hemiplegic lower limb. J Rehabil Med. 2004;36(4):159-64.

7. Shimodozono M, Noma T, Nomoto Y, Hisamatsu N, Kamada K, Miyata R, Matsumoto S, Ogata A, Etoh S, Basford JR, Kawahira K. Benefits of a repetitive facilitative exercise program for the upper paretic extremity after subacute stroke: a randomized controlled trial. Neurorehabil Neural Repair. 2013;27(4):296-305.

8. Bütefisch C, Hummelsheim H, Denzler P, Mauritz KH. Repetitive training of isolated movements improves the outcome of motor rehabilitation of the centrally paretic hand. J Neurol Sci. 1995;130(1):59-68.

9. Hoei T, Kawahira K, Fukuda H. Use of an arm weight-bearing combined with upper-limb reaching apparatus to facilitate motor paralysis recovery in an incomplete spinal cord injury patient: a single case report. J Phsyical Ther Sci. 2017:29:176-80.

10. Gheorghe L, Chavarriaga R, Millán JdeIR. Steering timing prediction in a driving simulator task. In: Proceedings of annual international conference of the IEEE engineering in medicine and biology society. IEEE engineering in medicine and biology society; 2013, p. 6913-16.

11. Zhang H, Chavarriaga R, Gheorghe L, Millan JDR. Inferring driver's turning direction through detection of error related brain activity. In: Proceedings of annual international conference IEEE Engineering Medicine Biol Soc. EMBS; 2013. p. 2196-99.

12. Khaliliardali Z, Chavarriaga R, Andrei Gheorghe L, Millan JDR. Detection of anticipatory brain potentials during car driving. In: Proceedings of annual international conference IEEE engineering medicine biologly society EMBS; 2012. p. 3829-32.

13. Choi K, Cichocki A. Control of a wheelchair by motor imagery in real time. In Intelligent data engineering and automated learning-IDEAL; 2008. p. $330-7$.

14. Nittono H. Event-related potentials (ERPs) and cognitive activity : a new perspective from engineering psychology, p. 1-19.

15. Pfurtscheller G, Lopes FH. Event-related EEG/MEG synchronization and desynchronization: basic principles. Clin Neurophysiol. 1999;110:1842-57.

16. Shibasaki H, Barrett G, Halliday E, Halliday AM. Components of the movement-related cortical potential and their scalp topography. Electroencephalogr Clin Neurophysiol. 1980;49(3-4):213-26.

17. Georgiev D, Lange F, Seer C, Kopp B, Jahanshahi M. Movement-related potentials in Parkinson's disease. Clin Neurophysiol. 2016;127(6):2509-19.

18. Suykens JAK, Vandewalle J. Least squares support vector machine classifiers. Neural Process Lett. 1999;9(3):293-300.

19. Tong S, Koller D. Support vector machine active learning with applications to text classification. J Mach Learn Res. 2001;2:45-66.

20. Furey TS, Cristianini N, Duffy N, Bednarski DW, Schummer M, Haussler D. Support vector machine classification and validation of cancer tissue samples using microarray expression data. Bioinformatics. 2000;16(10):906-14

21. Etoh S. Evaluation of cortical plasticity with TMS. Japanese J Rehabil Med. 2002:39(12):773-6.

\section{Submit your manuscript to a SpringerOpen ${ }^{\circ}$ journal and benefit from:} walking in adults with chronic hemiplegia. Arch Phys Med Rehabil. 2010;91(5):687-96.

3. Langhorne P, Coupar F, Pollock A. Motor recovery after stroke: a systematic review. Lancet Neurol. 2009;8(8):741-54.

4. Barnes MP. Principles of neurological rehabilitation. J Neurol Neurosurg Psychiatry. 2003;74(Suppl 4):iv3-7.

5. Shindo K, Kawashima K, Ushiba J, Ota N, Ito M, Ota T, Kimura A, Liu M. Effects of neurofeedback training with an electroencephalogram-based brain-computer interface for hand paralysis in patients with chronic stroke: a preliminary case series study. J Rehabil Med. 2011;43(10):951-7.
- Convenient online submission

$\checkmark$ Rigorous peer review

- Open access: articles freely available online

- High visibility within the field

- Retaining the copyright to your article

Submit your next manuscript at springeropen.com 